Revista Ibero-Americana de Ciências Ambientais

Ibero-American Journal of Environmental Sciences

Edição Especial do Congresso de Gestão Ambiental do Baixo Amazonas

(CONGABA) Ago 2018 - v.9 - n.6

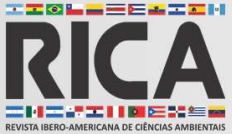

ISSN: 2179-6858

\title{
Caracterização biométrica e composição química do fruto de Caryocar villosum (Aubl.) Pers. nativo da Amazônia
}

Caryocar villosum (Aubl.). Pers (Caryocaraceae) é uma espécie da Amazônia de uso múltiplo, seja da madeira, seja dos frutos utilizados na alimentação, seja para obtenção do óleo. Objetivouse, com este estudo, realizar a caracterização física, avaliar a correlação dos dados e determinar a composição química de frutos de piquiá coletados em Piquiatuba, Floresta Nacional do Tapajós (FLONA), Belterra (PA). Em laboratório, mensurou-se o diâmetro longitudinal (DL), diâmetro transversal (DT), massa fresca (MF) do fruto, da casca, da polpa e do pirênio, rendimento da polpa (\%) e composição química (umidade, cinzas, proteínas, carboidratos e lipídeos). Os dados físicos foram analisados por distribuição de frequência e realizada correlação de Pearson (r), enquanto os dados de composição química foram expressos em média \pm desvio-padrão. Os frutos apresentaram DL e DT variando, respectivamente, entre 76 a $106 \mathrm{~mm}$ e 75 a $130 \mathrm{~mm}$. A (r), enquanto os dados de composição química foram expressos em média \pm desvio-padrão. Os frutos apresentaram DL e DT variando, respectivamente, entre 76 a $106 \mathrm{~mm}$ e 75 a $130 \mathrm{~mm}$. A
MF dos maiores frutos foi de $758,78 \mathrm{~g}$ e dos menores $262,45 \mathrm{~g}$. Já a MF da casca variou entre $158 \mathrm{~g}$ a $603 \mathrm{~g}$, enquanto a MF dos pirênios com polpa variou entre $61 \mathrm{~g}$ a $271 \mathrm{~g}$. A MF da polpa variou entre $31 \mathrm{~g} \mathrm{e} 91 \mathrm{~g}$, com maior frequência entre 41 e $51 \mathrm{~g}$, enquanto o rendimento da polpa variou entre $8 \%$ e $20 \%$. Verificou-se correlação positiva entre o diâmetro longitudinal (DL) e massa fresca (MF) da casca $(r=0,8421, p<0,05)$ MF do fruto e diâmetro transversal (DT) $(r=0,7264, p<0,05)$, DT e MF da casca $(r=0,7866, p<0,05)$, MF do fruto e MF da casca $(r=0,9169$ , $p<0,05)$, MF do pirênio e MF da polpa $(r=0,8626, p<0,05)$ e MF do fruto e MF da polpa $(r=0,6717, p<0,05)$. Sobre a análise de composição química, verificou-se que o piquiá é rico em lipídeos e carboidratos com, respectivamente, $23,65 \%$ e $26,74 \%$ de sua composição. Também representa uma boa fonte proteica com $15,69 \%$ e elevado grau de umidade com $32,55 \%$ de composição da polpa além de resíduo mineral fixo (cinzas) de 1,37\%. Com base nos resultados, considera-se que as características físicas dos frutos sugerem variabilidade das plantas de piquiá encontradas na área de coleta e a composição química potencial para aproveitamento da polpa como fonte de calorias, proteínas e minerais.

Palavras-chave: Piquiá; Composição Centesimal; Biometria.

\section{Biometric characterization and chemical composition of Caryocar villosum (Aubl.) Pers. native of the Amazon}

\begin{abstract}
Caryocar villosum (Aubl.). Pers (Caryocaraceae) is a multi-use species from the Amazon, either from wood, from fruits used for food, or to obtain oil. The objective of this study was to perform the physical characterization, to evaluate the correlation of the data and to determine the chemical composition of piquiá fruits collected in Piquiatuba, Tapajós National Forest (FLONA) Belterra (PA). In the laboratory, the longitudinal diameter (DL), transverse diameter (DT), fresh mass (MF) of the fruit, peel, pulp and pyrene, pulp yield (\%) and chemical composition (moisture, ash, proteins, carbohydrates and lipids). Physical data were analyzed by frequency distribution and Pearson correlation ( $r$ ) was performed, while chemical composition data were expressed ash, proteins, carbohydrates and lipids). Physical data were analyzed by frequency distribution and Pearson correlation ( $\mathrm{r}$ ) was performed, while chemical composition data were expressed
as mean \pm standard deviation. The fruits presented DL and DT varying, respectively, between 76 to $106 \mathrm{~mm}$ and 75 to $130 \mathrm{~mm}$. The FM of the largest fruits was $758,78 \mathrm{~g}$ and the smallest as mean \pm standard deviation. The fruits presented DL and DT varying, respectively, between 76 to $106 \mathrm{~mm}$ and 75 to $130 \mathrm{~mm}$. The FM of the largest fruits was $758,78 \mathrm{~g}$ and the smallest
$262,45 \mathrm{~g}$. The bark FM ranged from $158 \mathrm{~g}$ to $603 \mathrm{~g}$, while the pulp pyrenium FM ranged from $61 \mathrm{~g}$ to $271 \mathrm{~g}$. Pulp MF ranged from $31 \mathrm{~g}$ to $91 \mathrm{~g}$, most often between 41 and $51 \mathrm{~g}$, while pulp yield ranged from $8 \%$ to $20 \%$. Positive correlation was found between longitudinal diameter (DL) and fresh mass (MF) of rind $(r=0.8421, p<0.05)$ fruit MF and transverse diameter (DT) ( $r=0.7264$ $p<0.05)$, DT and shell MP $(r=0.7866, p<0.05)$, fruit MP and shell MP $(r=0.9169, p<0.05)$, pyrene MP and Pulp MF $(r=0.8626, p<0.05)$ and fruit MF and pulp MF ( $r=0.6717, p<0.05)$. Regarding the chemical composition analysis, it was found that piquiá is rich in lipids and carbohydrates with, respectively, $23,65 \%$ and $26,74 \%$ of its composition. It also represents a good protein source with $15,69 \%$ and high moisture content with $32,55 \%$ pulp composition as well as $1.37 \%$ fixed mineral residue (ash). Based on the results, it is considered that the physical characteristics of the fruits suggest variability of piquiá plants found in the collection area and the potential chemical composition for use of pulp as a source of calories, protein and minerals.
\end{abstract}

Keywords: Piquiá; Centesimal composition; Biometry.

Topic: Uso de Recursos Naturais

Reviewed anonymously in the process of blind peer
Received: $\mathbf{2 3 / 0 8 / 2 0 1 8}$

Approved: 28/08/2018
Carla Tatiane Borges Leandro

Universidade Federal do Oeste do Pará, Brasil http://lattes.cnpq.br/8717413476307778

carlatatianeborges@gmail.com

Elcio Meira Da Fonseca Junior (iD

Universidade Federal do Oeste do Pará, Brasil http://lattes.cnpq.br/6643016343021337 http://orcid.org/0000-0002-5025-7096

meirafonseca@yahoo.com.br

\section{Fabrizia Sayuri Otani}

Universidade Federal do Oeste do Pará, Brasi

http://lattes.cnpq.br/3506071373362378

fabrizia.otani@ufopa.edu.br
Cristina Aledi Felsemburgh

Universidade Federal do Oeste do Pará, Brasil

http://lattes.cnpq.br/8446110971099607

crisalefel@gmail.com
Referencing this:

LEANDRO, C. T. B.; FONSECA JUNIOR, E. M.; OTANI, F. S.; FELSEMBURGH, C. A.. Caracterização biométrica e composição química do fruto de Caryocar villosum (Aubl.) Pers. nativo da Amazônia. Revista Ibero-Americana de Ciências Ambientais, v.9, n.6, p.295-306, 2018. DOI: http://doi.org/10.6008/CBPC2179$\underline{6858.2018 .006 .0028}$ 


\section{INTRODUÇÃO}

As espécies conhecidas como pequizeiro e nomes derivados, Caryocaraceae, da ordem Theales (Rizobolacea), são compostas de 25 espécies reunidas em dois gêneros, Caryocar e Anthodiscus (PESSOA et al., 2015). O gênero Caryocar possui 16 espécies, das quais, doze são encontradas no território brasileiro (FRANCO et al., 2004), em sete estados (Acre, Amazonas, Amapá, Maranhão, Pará, Rondônia, Roraima), com variação na espécie encontrada em cada região (GIACOMETTI, 1993). Na Amazônia Oriental, destaca-se o Caryocar villosum (Aubl.). Pers., na região de caatinga do Nordeste, Caryocar cortaceum e Caryocar coriaceum e nas regiões sudeste e central, Caryocar brasiliense Camb. (MAIA et al., 2015; XAVIER et al., 2011; PESSOA et al., 2015).

Estudos realizados com $C$. brasiliense e $C$. villosum, mostram que as espécies desse gênero são fortemente alógamas (GRIBEL et al., 1993; MARTINS et al., 2007), onde pequenos morcegos nectarivoros (Glossophaga socicina e Anoura geoffroyi) são os principais polinizadores e a protandria e a hercogamia (separação espacial de anteras e estigmas) funcionam como principais mecanismos contra a autogamia (GRIBEL et al., 1993). Porém, a respeito da alogamia acentuada, a autopolinização pode ocorrer em pequena proporção (GRIBEL et al., 1993; MARTINS et al., 2007).

Em geral, a época de floração depende da região de ocorrência e as informações fenológicas descritas, mostram que a espécie é semidecídua, com redução parcial da folhagem durante a estação seca (CORNER, 1976). A floração ocorre logo após a emissão das folhas novas e os frutos alcançam a maturidade entre três e quatro meses após a floração com baixa taxa de vingamento de frutos (CORNER, 1976; ARAÚJO, 1995). Somado a isso, $20 \%$ a $30 \%$ das árvores de piquiá produzem frutos todos os anos, as demais descansam um ano produzindo no seguinte (OLIVEIRA et al., 2008; PINTO, 2007).

O C. villosum, árvore típica da Amazônia, de até 50 metros de altura e 2,5 metros de diâmetro (SHANLEY et al., 2005), teve sua origem provável na Amazônia Oriental e encontra-se distribuído por toda a floresta equatorial (CLAY et al., 1993). Esta árvore apresenta usos múltiplos, como para extração de madeira, consumo da polpa e extração de óleo do fruto (CARVALHO et al., 2006). A madeira é usada na produção de dormentes, tanoaria, na construção civil e naval (MARX et al., 1997; VESTANO JÚNIOR et al., 1983).

O fruto pode ser consumido após o cozimento, sendo o mesocarpo interno ou polpa a parte comestível (SOUZA, 2005), sendo utilizada da mesma forma que o pequizeiro (Caryocar brasiliense Camb.), em forma de pequizada, consumida em cozidos de carne de gado e de frango, no feijão, no arroz, e no prato regional baião de dois (LORENZO, 2017; CARVALHO et al., 2006; OLIVEIRA et al., 2008). Da polpa do fruto, é extraído o óleo que, além de utilizado na culinária, é empregado na indústria cosmética, na produção de sabão e como produto medicinal (PEIXOTO, 1973a).

As indicações sobre o uso do C. villosum como fármaco pelas populações tradicionais, são para tratamentos de doenças virais como gripes e em queimaduras (MORAES, 2011). Segundo GALUPPO (2004), a presença de compostos orgânicos, como esteroides e triterpenoides, caracterizam o óleo do pequi (Caryocar glabrum) como produto fitoterápico, medicamento com componentes terapêuticos derivados exclusivamente de plantas. 
O fruto de $C$. villosum é uma drupa e quando maduro, apresenta mesocarpo externo com coloração verde-clara a levemente amarelada (SHANLEY et al., 2005), contendo um ou dois pirênios, no entanto, é possível encontrar frutos com até quatro, sendo dois grandes e volumosos e dois pequenos; o epicarpo ou casca apresenta coloração marrom-esverdeada, o endocarpo é rígido e espinhoso, característico do gênero (FERREIRA et al., 1988). O mesocarpo interno (polpa) é oleoso, moderadamente grosso e denso, a cor pode variar de bege a amarelo vivo (CORNER, 1976). Os frutos variam de tamanho, com peso médio de 200g, verificados na região de Laranjal do Jari (AP) (BEZERRA et al., 2006).

No que se refere às características biométricas de $C$. villosum, poucas são as informações disponíveis. Para C. brasiliense, Ferreira et al. (1988) verificou que a casca do fruto maduro representa cerca de $84 \%$ do peso, a polpa $10 \%$ e a semente $6 \%$ do peso total. Bezerra et al. (2006) encontrou para C. villosum, respectivamente, $58,63 \%, 7,28 \%$ e $34,09 \%$, para casca, polpa e endocarpo; apresentando frutos com peso médio de 202,9g; comprimento 69,75mm; largura 83,92mm e apenas uma semente.

Semelhante à biometria, poucas informações são conhecidas quanto à composição química de $C$. villosum (piquiá). FAO (1986) relata para o mesocarpo de piquiá a composição de 3\% de proteínas; $14 \%$ de fibras, $11 \%$ de carboidratos e $31,1 \%$ de lipídeos segundo Marx et al. (1996). Pesquisas realizadas com C. brasiliense, que como frutífera é mais conhecida na região central do país, mostram que o mesmo pode apresentar diferenças na composição química, podendo ser oriunda da diversidade genética ou fenotípica entre frutos de regiões diferentes ou da mesma região de origem (ALVES et al., 2012; LIMA et al., 2007; OLIVEIRA et al., 2006; OLIVEIRA et al., 2009; VERA et al., 2007).

Além da carência de estudos com C. villosum, nenhuma das espécies de Caryocar foram domesticadas. Até mesmo C. brasiliense, mais explorada comercialmente, encontra-se em estágio intermediário de domesticação para plantas perenes, de tal modo que a exploração se dá prioritariamente, por meio do extrativismo (FRANCO et al., 2004; MENDES et al., 2016). Pela literatura consultada, os estudos disponíveis em sua maioria são com $C$. brasiliense, muitos dos quais poderão auxiliar na compreensão do $C$. villosum, em virtude da proximidade das duas espécies. A melhor compreensão da espécie poderá contribuir para a sua conservação, o aproveitamento sustentável dos frutos e geração de renda por grupos populacionais da Amazônia.

Além disso, é um produto indispensável na alimentação das populações que vivem ao redor das áreas de ocorrência da espécie, que fornece parte dos aportes energéticos e nutricionais necessários, principalmente para as famílias carentes, no período da safra (CLAY et al., 1993). Neste sentido, ressalta-se a importância de estudos sobre as características físicas e químicas dos frutos de $C$. villosum. $O$ objetivo do presente estudo, portanto, foi avaliar e correlacionar características biométricas, e determinar a composição química de frutos de $C$. villosum do Baixo Amazonas. 


\section{MATERIAL E MÉTODOS}

\section{Local de coleta dos frutos}

O local de coleta dos frutos foi a Floresta Nacional (Flona) do Tapajós, na comunidade de Piquiatuba, Belterra (PA). A Flona do Tapajós localiza-se entre os paralelos de $2^{\circ} 45^{\prime}$ e $4^{\circ} 10^{\prime}$ de latitude sul e entre os meridianos de $54^{\circ} 45^{\prime}$ e $55^{\circ} 30^{\prime}$ de longitude oeste, limitando ao sul com a Rodovia Transamazônica e ao norte, com a rodovia Cuiabá-Santarém (BR-163); e os Rios Cupari e Cuparitinga ou Santa Cruz; a leste, com a BR-163; e a oeste com o Rio Tapajós, com área estimada de 600.000ha (ESPÍRITO-SANTO et al., 2015). A comunidade de Piquiatuba abrange 2.153 hectares da Flona, sendo localizada na margem direita do rio Tapajós, município de Belterra (IBAMA, 2004), entre as comunidades de Pedreira e Marituba.

A vegetação da Flona Tapajós é caracterizada por ser de Floresta Ombrófila Densa (IBAMA, 2004). O clima da região segundo a classificação de Köppen é do tipo Ami (IBAMA, 2004) com temperatura média anual superior a $26^{\circ} \mathrm{C}$. A precipitação média anual está entre 1.900 e $2.400 \mathrm{~mm}$, com regime de chuvas apresentando grande variação durante o ano, com as maiores precipitações ocorrendo nos meses de janeiro a maio (ALVARES et al., 2014).

Para o presente estudo, as árvores foram amostradas conforme estudo realizado previamente por este grupo de pesquisa na Comunidade de Piquiatuba (LIMA et al., 2018) em duas áreas de 250ha com 25

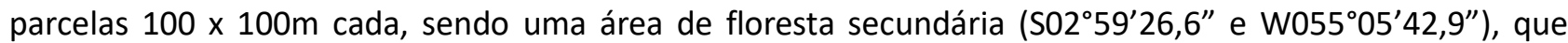
atualmente ocorre extrativismo de frutos de $C$. villosum, sendo mais próximo aos comunitários de Piquiatuba em relação a segunda área. A segunda área é de floresta primária $\left(S 03^{\circ} 00^{\prime} 28,4^{\prime \prime}\right.$ e W055 $\left.02^{\prime} 38,1^{\prime \prime}\right)$, sem uso extrativista, onde se observa a predominância de árvores de grande porte com dossel mais fechado, se comparado com a primeira área. Por ser mais próximo da comunidade de Piquiatuba e conter maior número de indivíduos, foi realizada a coleta na primeira área.

Nesta área foram selecionados os indivíduos adultos e com produção de frutos no momento da coleta. Foram coletados, separadamente, trinta e cinco frutos de três plantas matrizes, diretamente do chão, em abril de 2015, totalizando 105 frutos. Os frutos foram acondicionados em sacos plásticos com fechamentos herméticos e levados ao Laboratório de Tecnologia de Produtos de Origem Animal (TPOA) da Universidade Federal do Oeste do Pará (UFOPA). No laboratório, os frutos de cada planta matriz foram selecionados separando-se aqueles visualmente sadios, inteiros e sem deformação. Os indivíduos foram georreferenciadas com auxílio de GPS marca Garmin, modelo 76CSx: indivíduo 1 (S 02 97'900" e W $055^{\circ}$

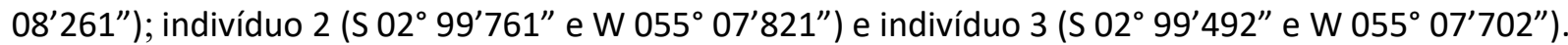

\section{Caracterização física}

Em laboratório, foram selecionados 80 frutos sadios, isto é, inteiros e sem deformação, sendo 20 de cada matriz, e processados imediatamente. Os frutos foram classificados quanto ao tamanho (diâmetro longitudinal e transversal), e determinada a massa fresca (MF). Em seguida foram descascados manualmente, com facas de aço inoxidável, para a separação dos pirênios da casca e obtenção da polpa para a posterior 
determinação da massa dos pirênios, massa da polpa e massa da casca. O diâmetro foi determinado com auxílio de um paquímetro digital e a massa utilizando-se uma balança semianalítica.

\section{Caracterização química: preparo das amostras}

Para as análises de composição química, frutos de piquiá provenientes das três matrizes selecionadas (10, 13 e 14), foram descascados e despolpados manualmente, com auxílio de faca de aço inoxidável. Em seguida, a polpa do fruto foi triturada e homogeneizada, acondicionada em sacos plásticos com fechamento hermético, e armazenada em freezer doméstico, a $-18^{\circ} \mathrm{C}$, para as análises. Todas as análises de composição química foram feitas de cada amostra das três matrizes, em triplicata.

\section{Determinação da composição química}

O teor de umidade foi determinado pelo método gravimétrico em estufa a $105^{\circ} \mathrm{C}$, até peso constante, segundo as normas do (IAL, 2008); o resíduo mineral fixo por incineração em mufla à $550^{\circ} \mathrm{C}$ (CASTRO et al., 2014); o teor de nitrogênio pelo método de micro-kjeldahl e convertido em proteína bruta pela multiplicação do resultado ao fator 6,25 (AOAC, 1990); os lipídios totais, extraídos por meio da técnica de Bligh et al. (1959), e posteriormente determinados por gravimetria. Os carboidratos foram determinados por diferença, subtraindo-se de 100 os valores obtidos para umidade, proteínas, lipídios e resíduo mineral fixo.

\section{Análise dos dados}

Os dados de biometria foram analisados mediante distribuição de frequência. Foi calculado o coeficiente de correlação de Person ( $r$ ), e o respectivo nível de significância entre as variáveis. As características biométricas foram analisadas pelo teste de Lilliefors, para verificação da normalização de sua distribuição (ZAR, 1999). Para a composição química, os resultados obtidos foram expressos em média \pm desvio-padrão, sendo a estatística descritiva calculada utilizando o programa estatístico SAS ${ }^{\circledR}$, versão 9.3 .

\section{RESULTADOS}

Os frutos da população analisada apresentaram diâmetro longitudinal (DL) médio de $87,9 \mathrm{~mm}$, variando entre 76 a $106 \mathrm{~mm}, 42 \%$ dos frutos apresentaram DL entre 86 e $91 \mathrm{~mm}$ (figura 1A). 0 diâmetro transversal (DT) médio de 89,7mm, variando entre 75 a 130mm (figura 1A), com $40 \%$ dos frutos com DT de 75 a $87 \mathrm{~mm}$ e $42 \%$ de 87 a $99 \mathrm{~mm}$ (figura $1 \mathrm{~B}$ ).

Os frutos apresentaram média de massa de $437,45 \mathrm{~g}$, sendo que os maiores chegaram a ter massa de $758,78 \mathrm{~g}$ e os menores $262,45 \mathrm{~g}$ (figura $1 \mathrm{C}$ ). A massa da casca representou a maior porção dos frutos com $62 \%$, onde $32 \%$ dos frutos apresentando massa de casca entre 232 a 306g, 30\% entre 306 a 380g, 17\% entre 158 a $232 \mathrm{~g}, 17 \%$ entre 380 a $354 \mathrm{~g}, 3 \%$ entre 454 a $528 \mathrm{~g}$ e $2 \%$ entre 358 a $602 \mathrm{~g}$ (figura 1D). 


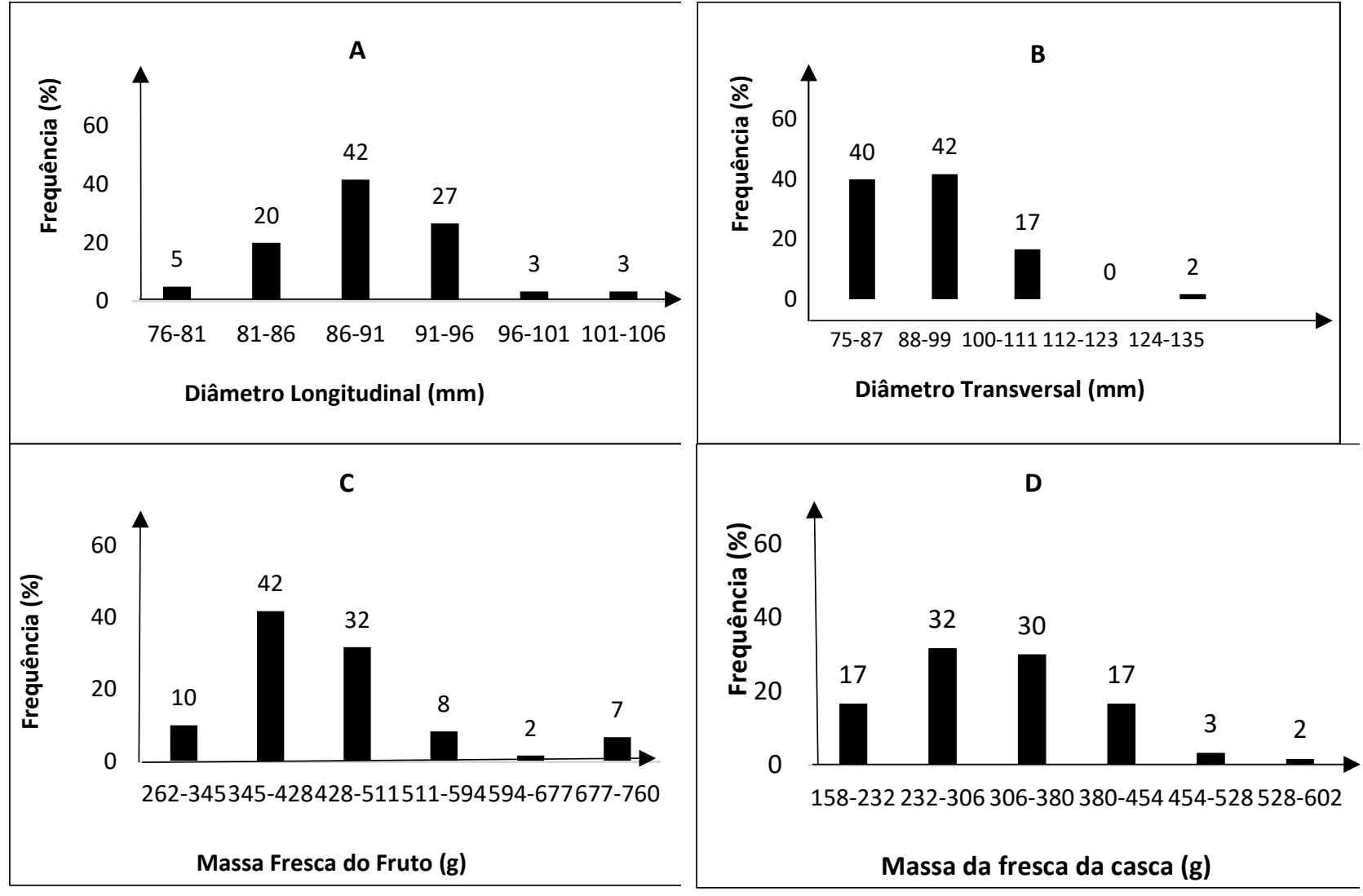

Figura 1: Caracterização biométrica de frutos de C. villosum da Floresta Nacional do Tapajós, comunidade de Piquiatuba, Belterra (PA): (A) Diametro tranversal; (B) Diametro longitudinal; (C) Massa fresca do fruto; e (D) Massa fresca da casca

A maioria dos frutos apresentaram massa da polpa entre 41 e $51 \mathrm{~g}$, correspondendo a $50 \%$ dos frutos (figura $2 \mathrm{~A}$ ) e rendimento da polpa com maiores frequências variando entre 10 e $12 \mathrm{~g}$, 12 e $14 \mathrm{~g}$ e 14 e $16 \mathrm{~g}$ correspondendo à $28 \%, 25 \%$ e $30 \%$, respectivamente (figura 2B). Já a massa dos pirênios com polpa representou $38 \%$ do total, variando entre $61 \mathrm{~g}$ a $271 \mathrm{~g}$, sendo os maiores valores encontradas entre 61 a $96 \mathrm{~g}$, 96 a 131g, 131 a 166g o que corresponde a uma frequência de 30, 38 e 20 \% (figura 2C).

Da análise de correlação entre as variáveis, verificou-se associação positiva e significativa entre: diâmetro longitudinal (DL) e massa fresca (MF) da casca $(r=0,8421$, $p<0,05)$ MF do fruto e diâmetro transversal (DT) $(r=0,7264, p<0,05)$, DT e MF da casca $(r=0,7866, p<0,05)$, MF do fruto e MF da casca $(r=$ $0,9169$, $p<0,05)$, MF do pirênio e MF da polpa $(r=0,8626$, $p<0,05)$ e MF do fruto e MF da polpa $(r=0,6717$ , $\mathrm{p}<0,05)$. Por outro lado, não se correlacionaram significativamente as variáveis: MF da casca e MF do pirênios ( $r=0,2577, p<0,05)$ e MF da casca e MF da polpa $(r=0,3917, p<0,05)$. Da análise da composição química, verificou-se que a polpa dos frutos de piquiá apresenta teor de lipídeos de 23,65\%, proteínas de 15,69\%, carboidratos de $26,74 \%$, resíduo mineral fixo (cinzas) de $1,37 \%$ e teor de umidade correspondendo a $32,55 \%$ (tabela 1 ).

Tabela 1: Composição química da polpa de frutos de piquiá, da Floresta Nacional do Tapajós, comunidade de Piquiatuba, Belterra (PA).

\begin{tabular}{|l|l|}
\hline Constituintes químicos & Média \pm desvio padrão (\%) \\
\hline Lipídios & $23,65 \pm 4,0$ \\
\hline Proteínas & $15,69 \pm 5,3$ \\
\hline Cinzas & $1,37 \pm 2,5$ \\
\hline Umidade & $32,55 \pm 4,0$ \\
\hline Carboidratos & $26,74 \pm 4,0$ \\
\hline
\end{tabular}



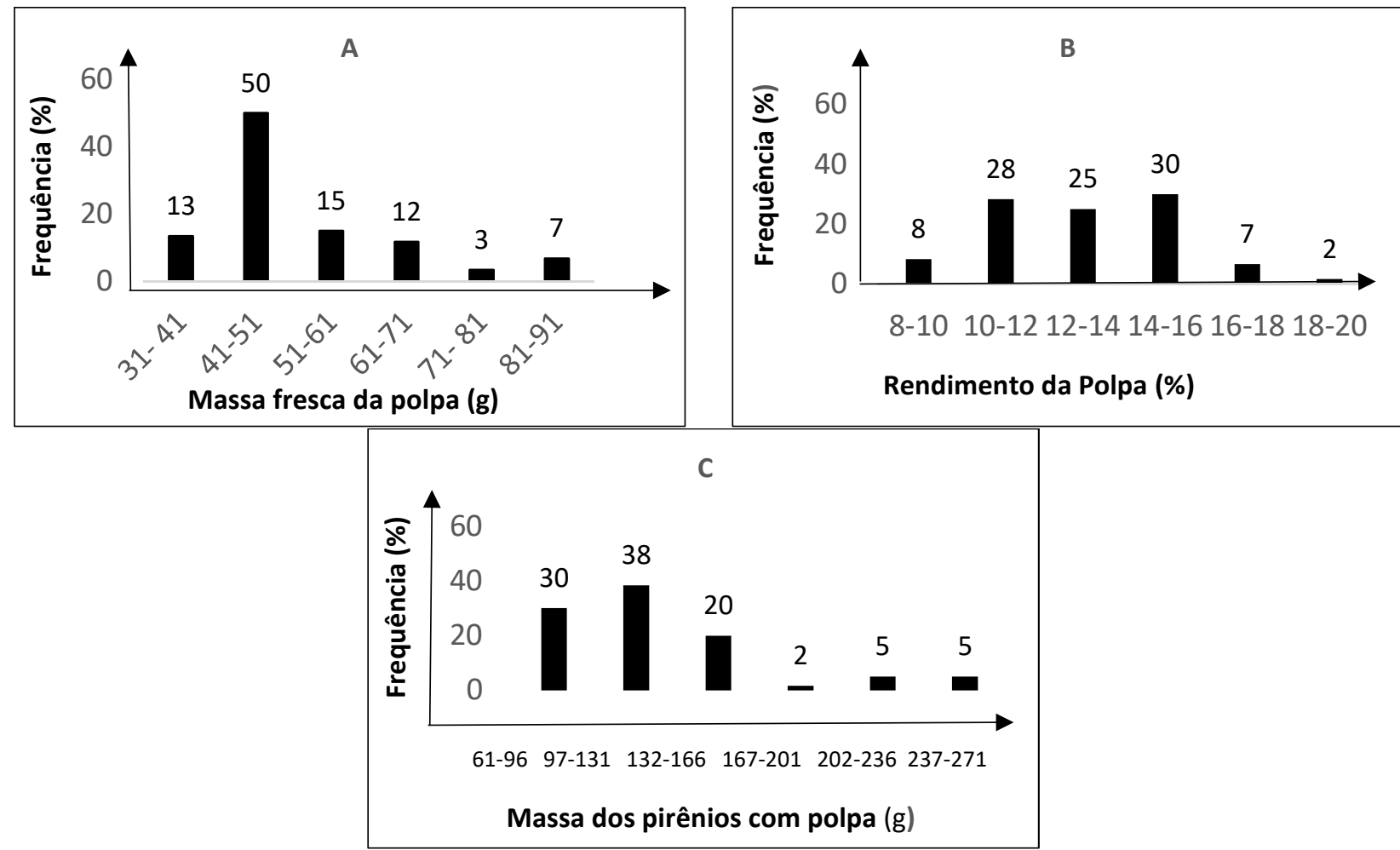

Figura 2: Caracterização biométrica da polpa de frutos de $C$. villosum da Floresta Nacional do Tapajós, comunidade de Piquiatuba, Belterra-PA: (A) Massa fresca da polpa, (B) Rendimento da polpa e (C) Massa dos pirênios com polpa.

\section{DISCUSSÃO}

Os estudos referentes à caracterização biométrica de frutos constituem importante ferramenta para o entendimento de formas de exploração comercial dos frutos, permitem a determinação de parâmetros de qualidade e rendimento, além de subsidiarem o dimensionamento de máquinas, equipamentos e programas de melhoramento genético das espécies (OLIVEIRA et al., 2009). As dimensões de diâmetro transversal (DT) e longitudinal (DL) encontradas neste estudo para frutos de piquiá da comunidade de Piquiatuba (DT: $89,71 \mathrm{~mm}$ e DL $87,90 \mathrm{~mm}$ ) foram maiores que as relatadas por Bezerra et al. (2006) para Caryocar villosum, no município de Laranjal do Jari (AP), onde o mesmo relatou, DT e DL médio de $83,92 \mathrm{~mm}$ e $69,75 \mathrm{~mm}$, variando entre 70,33 a 108,71 mm e 64,78 a $75,91 \mathrm{~mm}$, respectivamente.

De modo semelhante, os frutos analisados no presente estudo, apresentaram massa fresca média de 437,45g, com 42\% dos frutos com massa entre 345 a 428g, superior à média de 202g encontrada por estes autores. De modo análogo, a massa fresca média de frutos de $C$. villosum, de $437,45 \mathrm{~g}$, foi superior àquela verificada por Ramos et al. (2011), em frutos de C. coreaceum do Maranhão e Piauí, respectivamente, de 142, 76g e 190,3g, e de C. brasiliense do Mato Grosso com massa média de frutos de 158g (CORDEIRO et al., 2013) e de São Miguel do Araguaia (GO), com massa dos frutos variando de 81,59g a 198,13g (SOARES JUNIOR et al., 2010).

O mesocarpo interno ou polpa, a parte comestível do fruto, representou $11 \%$ do total do fruto, apresentando massa média de 51,49g, ou seja, a menor porção (figura $2 \mathrm{~A}$ ), valor esse 3,5 vezes superior aquele encontrado por Bezerra et al. (2006) de 14,77g para a mesma espécie no Amapá. A proporção de massa fresca da casca, pirênio e polpa em relação a massa total do fruto neste estudo foi similar ao que Peixoto (1973) descreveu para frutos de C. brasiliense Camb., equivalentes a 65\%, 35\% e 11\%, porém, 
diferindo do verificado por Cordeiro et al. (2013) com frutos de C. brasiliense de Mato Grosso em que a casca representou $80 \%$ do peso dos frutos, pirênios $12 \%$ e a polpa $8 \%$.

Os resultados da análise de correlação evidenciaram forte associação entre a MF do fruto e MF da casca $(r=0,917)$ indicando que frutos com maior MF da casca são mais pesados A correlação entre a MF do fruto e MF da polpa foi de $r=0,672$, ou seja, a MF do fruto é diretamente proporcional a quantidade de polpa que em média contribui com $12 \%$ da MF total do fruto demonstrando um bom rendimento de polpa para frutos coletados na Flona do Tapajós, comunidade de Piquiatuba.

Observou-se ainda correlação positiva entre a MF do pirênio e a MF da polpa $(r=0,862 ; p<0,05)$, ou seja, pirênios maiores apresentam maior quantidade de polpa, diferindo dos resultados de Cordeiro (2012) para C. brasiliense, em que verificou uma baixa correlação positiva entre as massas dos frutos inteiros e as massas dos pirênios $(r=0,17)$, as massas de polpa $(r=0,28)$ e a altura dos frutos $(r=0,44)$, essas informações tratam-se de aspecto relevante visto que a massa dos frutos constitui-se parâmetro determinante para aquisição dos mesmos.

Sobre composição química da polpa dos frutos de piquiá, o teor de umidade encontrado de $32,55 \%$ foi menor que o relatado para C. brasiliense por LIMA (2007), de 41,40 \%, para frutos de Santa Rosa, no estado do Piauí, e por CORDEIRO (2013) de 50,62\% de Várzea Grande (MT). Por outro lado, os resultados encontrados neste estudo foram semelhantes aos encontrados para Caryocar coriaceum dos estados do Piauí e Maranhão que variaram de $25,21 \%$ a 37,91\% com média de 31,51\% (RAMOS et al., 2011). 0 teor de umidade é uma característica importante para conservação, já que elevados teores resultam na redução do tempo de prateleira e no comprometimento da qualidade, favorecendo a proliferação de microrganismos (RAMOS et al., 2011), por outro lado, a umidade está relacionada à suculência dos alimentos (KAJIYAMA et al., 2008), o que pode favorecer a aceitação do consumidor.

Quanto ao resíduo mineral fixo, o valor encontrado neste estudo de 1,37\% foi, aproximadamente, duas vezes maior que o encontrado por Lima et al. (2007), em frutos C. brasiliense, com valores de 0,63\%, e superior ao que Berto et al. (2015) encontrou para C. villosum da Amazônia (0,45\%). Esses resultados indicam que os frutos de piquiá da Flona do Tapajós, comunidade de Piquiatuba, são ricos em macro e micronutrientes com potencial aproveitamento na dieta alimentar.

Com relação aos lipídeos, verificou-se valores de $23,65 \%$, indicando que a polpa do fruto é altamente energética. Esses valores são próximos aos encontrados por Lima et al. (2007) na polpa de frutos de Caryocar brasiliense, com 22,52\% e inferiores aos verificados por Ramos et al. (2011) em frutos de C. coriaceumn de diferentes municípios dos estados de Maranhão e Piauí, apresentando valores de 34,78\% e 32,77 \%. Um dos principais subprodutos dos frutos do gênero Caryocar é o óleo (MENDES et al., 2016). Maiores teores de lipídeos nos frutos são interessantes para a extração do óleo e como fonte de ácidos graxos na alimentação dos consumidores; no entanto, maiores teores de lipídeos associados à umidade, tornam os frutos altamente perecíveis, tanto por reações de oxidação lipídica, assim como por proliferação de microrganismos.

Quanto aos teores de proteínas, os frutos de piquiá da comunidade de Piquiatuba apresentaram 15,69\%, superior ao descrito por Bezerra et al., (2006) em frutos de piquiá no município de Laranjal do Jari- 
AP (4,53\%). Esse valor proteico também é superior ao encontrado na polpa de $C$. brasiliense (3,09\%), proveniente de Goiânia (CORDEIRO, 2012). Os teores de proteínas encontrados neste estudo de 15,69\% são superiores aos encontrados em amêndoas de C. brasiliense, de 6,32\% (SOARES JÚNIOR et al., 2010), mas semelhante aos 13,5\% (OLIVEIRA et al., 2009) e inferior aos 25,27\% (LIMA et al, 2007) na polpa de frutos desta espécie. As proteínas consumidas por meio de alimentos, fornecem aos consumidores aminoácidos essenciais.

Os teores de carboidratos encontrados na polpa de piquiá $(26,74 \%)$ é superior ao que LIMA et al. (2007) verificaram para o C. brasiliense (11,45\%) do Piauí e de 7,02\% de Minas Gerais (RIBEIRO, 2011). No entanto, esse valor é inferior ao descrito na Tabela de Composição de Alimentos da Amazônia (AGUIAR, 1996) de $30,40 \%$ para o piquiá, e de 59,93\% verificado para C. coreaceum de Afonso Cunha (MA) (RAMOS, 2010).

De modo geral, os frutos de $C$. villosum da Flona do Tapajós apresentam características biométricas e composição química da polpa superiores àquelas verificadas para $C$. coriaceum, $C$. brasiliense e $C$. villosum do Amapá. O piquiá por se tratar de um alimento rico em minerais e com teores aceitáveis de lipídeos, proteínas e carboidratos pode ser considerado uma fonte alternativa de grande importância na alimentação humana, especialmente para as populações da Amazônia. O mesocarpo interno, parte comestível do fruto, desempenha importante parte da dieta dos nativos da região Amazônica (FAO, 1986), como é o caso do baixo Rio Tapajós, Oeste do Pará (ALVES et al., 2016).

A castanha presente no interior do endocarpo duro e espinhento, embora disponível e ter um bom sabor, é normalmente ignorada devido dificuldade de removê-la sendo o mesocarpo interno a principal fonte de alimento (FAO, 1986). Em concordância, Alves et al. (2016), em entrevista realizada com trinta e três informantes sobre os usos do piquiá, nas comunidades de Maguari e Jamaraquá na Flona do Tapajós, verificaram que o consumo da polpa do fruto após o cozimento do pirênio representa o principal uso do fruto seguido pelo óleo com fins medicinais.

Quanto à composição química da polpa do piquiá encontrada neste estudo se adequa às necessidades nutricionais diárias de um homem adulto (BRASIL, 2006). A necessidade diária de gorduras totais deve ser entre 15 a 30\% (BRASIL, 2006), estando dentro do indicado os teores de lipídeos encontrados nesse estudo, de 23,65\%. De modo semelhante, a ingestão diária de proteína indicada deve ser entre 10-15\% (BRASIL, 2006), estando dentro do indicado o encontrado neste estudo para C. villosun (15,69\%), no entanto, diferente do teor de proteínas encontrado por Bezerra et al. (2006) (4,53\%). 0 teor de carboidratos para consumo diário, segundo BRASIL (2006), é de 55 a 75\%, superior ao que encontramos neste estudo de $26,74 \%$. Com base nessa comparação, percebe-se que mesocarpo interno dos frutos de piquiá apresentam boa qualidade nutricional sendo indicado e de grande importância para alimentação das populações da Amazônia.

Uma outra informação importante, refere-se as características superiores para frutos de piquiá da Flona do Tapajós verificadas neste estudo que podem estar associadas à fatores ambientais tais como o tipo de vegetação, solo e clima da Amazônia ser bem diferente do Cerrado em que se localizam as espécies $C$. 
coriaceum e $C$. brasiliense. Essas espécies do Cerrado sobrevivem em locais extremamente pobres em nutrientes de solo e com elevado teor de alumínio tóxico e baixa precipitação (NAVES, 1999).

A Região Amazônica, por outro lado, caracteriza-se por elevada precipitação sendo o clima da região classificado do tipo Ami, segundo Köppen (IBAMA, 2004) apesar da Flona do Tapajós, Belterra (PA), ocorrer um período sazonal significativamente chuvoso (janeiro-junho) e outro menos chuvoso (julho a dezembro) bem definido (ESPÍRITO-SANTO et al., 2005). Em estudo realizado por nosso grupo de pesquisa sobre a fenologia com os mesmos indivíduos de piquiá, na Comunidade de Piquiatuba-Flona do Tapajós, verificou-se que o início do período chuvoso em outubro coincide com o início da frutificação e a maturação, de março a maio, com o período de máxima precipitação.

Considerando que a frutificação do piquiá de outubro a maio na área de estudo coincide com o período chuvoso, colabora para justificar frutos maiores e com composição química superior em relação à verificada para as espécies de Caryocar do Cerrado, embora fatores genéticos intrínsecos da espécie não devem ser descartados. Colaborando com esta hipótese, Castro Neto et al. (2003) observaram que o déficit hídrico durante o crescimento do fruto de manga (Mangifera indica (L.) reduz o acúmulo de massa seca e fresca do fruto. Adicionalmente, Léchaudel et al. (2007), em sua revisão de literatura, salienta que fatores ambientais como luz, temperatura, disponibilidade de água e de carbono afetam o crescimento do fruto de manga.

Neste contexto, as informações obtidas neste estudo podem ser relevantes para domesticação e melhoramento genético da espécie. Além de estudos das características físicas e químicas dos frutos poderem auxiliar no aproveitamento, assim como no desenvolvimento de tecnologias de conservação e processamento do piquiá.

\section{CONCLUSÕES}

Conclui-se que os frutos apresentaram alta variação nas medidas de MF e tamanho dos frutos na área de estudo, indicativo de possível ganho genético por meio de seleção das características agronômicas. A MF da polpa apresenta relação forte e positiva com MF do pirênio, MF da casca e MF do fruto. O conteúdo de resíduo mineral fixo encontrado nos frutos, sugere potencial de aproveitamento da polpa do piquiá como fonte de minerais.

Os altos teores de umidade e lipídeos são interessantes para suculência do fruto e extração de óleo, no entanto tornam a polpa de piquiá bastante propícia às deteriorações microbiológicas e rancidez do óleo, com influência direta nas características químicas e sensoriais dos frutos. Os teores de lipídeos, proteínas e carboidratos do piquiá indicam que o fruto pode ser utilizado como fonte de aminoácidos, ácidos graxos e calorias na alimentação humana, em vista de o carboidrato ser a principal fonte de calorias da dieta humana.

\section{REFERÊNCIAS}

AGUIAR, J. P. L.. Notas e comunicações: Tabela de Composição de Alimentos da Amazônia. Acta Amazônica, Manaus, v.26, n.2, p.121-126, 1996.
ALVARES, C. A.; STAPE, J. L.; SENTELHAS, P. C.; GONÇALVES, J. L. M.; SPAROVEK, G.. Köppen's climate classification map for Brazil. Meteorologische Zeitschrift, v.22, n.6, p.711-728, 2014. 
ALVES, A. M.; FERNANDES, D. C.; SOUSA, A. G. O.; NAVES, M. M. V.. Caracterização física e química de frutos do pequizeiro oriundos de três estados brasileiros. Ciência Rural, Santa Maria, v.38, n.6, p. 1790-1796, 2012.

ALVES, R. P; LEVIS, C.; CLEMENT, C. R.. Use and management of piquiá suggest in situ domestication along the lower Tapajós River, Brazilian Amazonia. Economic Botany, Bronx, v.70, n.2, 2016

AOAC. Association of Official Analytical Chemists. Official methods of analysis. $10 \mathrm{ed}$. Washington: AOAC, 1990.

ARAUJO, F. D.. A review of Caryocar brasiliense (Caryocaraceae) in economically valuable species of the central brazilian cerrados. Economic Botany, Bronx, v.49, n.1, p.40-48, 1995.

BERTO, A.; SILVA, A. F.; VISENTAINER, J. V.; MATSUSHITA, M.; SOUZA, N. E.. Proximate compositions, mineral contents and fatty acid compositions of native Amazonian fruits. Food Research International, Burlington, v.77, p.441-449, 2015.

BEZERRA, V. S.; PEREIRA, S. S. C.; FERREIRA, L. A. M.. Avaliações Físico-químicas do mesocarpo do piquiá (Caryocar villosum (Aubl.) Pers.). In: CONGRESSO BRASILEIRO DE PLANTAS OLEAGINOSAS, ÓLEOS, GORDURAS E BIODISEL, 3. Anais. Lavras: UFLA, 2006.

BLIGH, E. G.; DYER, W. J.. A rapid method of total lipid extraction and purification. Canadian Journal of Biochemistry and Physiology, Ottawa, v.37, n.8, p.911-917, 1959.

BRASIL. Portaria Interministerial no 66, de $\mathbf{2 5}$ de agosto de 2006. Altera os parâmetros nutricionais do Programa de Alimentação do Trabalhador - PAT. Brasília: MTE, 2006

CARVALHO, J. E. U.; NASCIMENTO, W. M. O.; MULLER, C. H.. Propagação do Pequiazeiro. Belém: Embrapa, 2006.

CASTRO NETO, M. T.; REINHARDT, D. H.. Relações entre parâmetros de crescimento do fruto da manga cv. HADEN. Revista Brasileira de Fruticultura, Jaboticabal, v.25, n.1, p.36-38, 2003.

CASTRO, D. S; SOUSA, E. P.; NUNES, J. S.; SILVA, L. M. M.; MOREIRA, I. S.. Caracterização física e físico-química de polpa de buriti (Mauritia flexuosa). Revista Verde de Agroecologia e Desenvolvimento Sustentável, Pombal, v.9, p.117-120, 2014.

CLAY, J. W.; CLEMENT, C. R. Selected species and strategies to enhance income generation from Amazonian forests. Roma: FAO, 1993.

CORDEIRO, M. W. S.. Caracterização física e química de frutos do pequizeiro (Caryocar brasiliense Camb.) de diferentes regiões do estado do Mato Grosso. Dissertação (Mestrado) - Universidade Federal do Goiás, Goiânia, 2012.

CORDEIRO, M. W. S.; CAVALLIERE, A. L. F.; FERRARI, P. H.; NAVES, M. M. V.. Características físicas, composição químico-nutricional e dos óleos essenciais da polpa de Caryocar brasiliense nativo do estado de Mato Grosso. Revista Brasileira de Fruticultura, Jaboticabal, v.35, n.4, p.1127-11139, 2013.
CORNER, E. J. H.. The seeds of dicotyledons. Cambridge University Press: Cambridge, 1976.

ESPÍRITO-SANTO, F. D. B.; SHIMABUKURO, Y. E; ARAGÃO, L. E. O. C.; MACHADO, E. L. M.. Análise da composição florística e fitossociológica da floresta nacional do Tapajós com o apoio geográfico de imagens de satélites. Acta Amazônica, v.35, n.2, p.155-173, 2005.

FAO. Food and Agriculture Organization of the United Nations. Food and fruit bearing forest species. Examples from Latin America. Rome: FAO, 1986.

FERREIRA, F. R.; BIANCO, S.; DURIGAN, J. F.; BELINGIERI, P. A.. Caracterização física e química de frutos maduros de pequi. In: CONGRESSO BRASILEIRO DE FRUTICULTURA. Anais. Campinas: SBF, 1988.

FRANCO, L. M. L.; UMMUS, M. E.; LUZ, R. A.. A distribuição do pequi (Caryocar brasiliense) na estação ecológica de Itirapina, SP. In: CONGRESSO BRASILEIRO DE GEÓGRAFOS. Anais. Goiânia: AGB, 2004.

GALUPPO, C. S.. Documentação do uso e valorização do óleo de piquiá (caryocar villosum (abul) pers.) e do leite do amapá-doce (brosimum parinarioedis ducke) para a comunidade de piquiatuba, floresta nacional do tapajós: estudos físicos, químicos, fitoquímicos e farmacológicos. Dissertação (Mestrado em Ciências Florestais) - Universidade Federal Rural da Amazônia. Belém, 2004.

GIACOMETTI, D. C.. Recursos genéticos de fruteiras nativas do Brasil. In: SIMPÓSIO NACIONAL DE RECURSOS GENÉTICOS DE FRUTEIRAS NATIVAS. Anais. Cruz das Almas: EMBRAPA, 1993.

GRIBEL, R.; HAY, J. D.. Pollination ecology of Caryocar brasiliense (Caryocaraceae) in Central Brazil cerrado vegetation. Journal of Tropical Ecology, Cambridge, v.9, p.199-211, 1993.

IAL. Instituto Adolfo Lutz. Métodos físico-químicos para análises de alimentos. 4 ed. São Paulo: IAL, 2008.

IBAMA. Instituto Brasileiro do Meio Ambiente e dos Recursos Naturais e Renováveis. Floresta Nacional do Tapajós: plano de manejo. Belterra: IBAMA, 2004.

KAJIYAMA, T.; PARK, K. J.. Influência da umidade inicial da alimentação no tempo de secagem em secador atomizador. Revista Brasileira de Produtos Agroindustriais, Campina Grande, v.10, n.1, p.1-8, 2008.

LÉCHAUDEL, M.; JOAS, J.. An overview of preharvest factors influencing mango fruit growth, quality and postharvest behavior. Brazilian Journal of Plant Physiology, v.19, n.4, p.287-298, 2007.

LIMA, A.. Composição química e compostos bioativos presentes na polpa e na amêndoa do pequi (Caryocar brasiliense Camb.). Revista Brasileira de Fruticultura, Jaboticabal, v.29, n.3, p.695-698, 2007.

LIMA, M. C.; COUCEIRO, S. R. M.; FONSECA JUNIOR, E. M. Estrutura etária e espacial de Caryocar villosum (Aubl.) Pers. na Floresta Nacional do Tapajós, Oeste do Pará. SEMINÁRIO 
DE PESQUISAS DA FLORESTA NACIONAL DO TAPAJÓS, 3.

Anais. SANTARÉM: ICMBio, 2018.

LORENZO, N. D.. Mesocarpo do pequi (Caryocar villosum Albl. Pers.): Incorporação em formulação de chocolate com vista a agregação de valor nutricional. Dissertação (Mestrado em Farmácia) - Universidade de São Paulo, São Paulo, 2017.

MAIA, J. G. S.; ANDRADE, E. H. A.; SILVA, M. H. L. Aroma volatiles of pequi fruits (Caryocar brasiliense Camb.) aqueous extract quality processed by membranes. Food and Bioproducts processing, Rugby, v.95, p.304-312, 2015.

MARTINS, R. L.; GRIBEL, R.. Polinização de Caryocar villosum (Aubl.) Pers. (Caryocaraceae) uma árvore emergente da Amazônia Central. Revista Brasileira de Botânica, São Paulo, v.30, n.1, p.37-45, 2007.

MARX, F.; ANDRADE, E. H. A.; MAIA, J. G.. Chemical composition of the fruit pulp of Caryocar villosum. $\mathbf{Z}$ Lebensm Unters Forsch, v.204, p.442-444, 1997.

MENDES, M. F.; NEVES, S. M. A. S.; SILVA, M. A.; PAIVA, S. L. P.; KREITLOW, J. P.. Coleta, processamento, comercialização e sustentabilidade da produção extrativista da região sudoeste Mato Grossense, Brasil. Revista Ibero-Americana de Ciências Ambientais, Aracaju, v.7, n.3, p.59-71, 2016.

MORAES, R. P.. Conservação socioambiental do piquiá (Caryocar villosum (Aubl.) Pers.) na região dos lagos Parú e Calado. Dissertação (Mestrado em Ciências do Ambiente e Sustentabilidade da Amazônia) -Universidade Federal do Amazonas, Manaus, 2011.

NAVES, R. V.. Espécies frutíferas nativas dos cerrados de Goiás: caracterização e influências do clima e dos solos. Tese (Doutorado em Agronomia) - Universidade Federal de Goiás, Goiânia, 1999.

OLIVEIRA, M. E. B.; GUERRA, N. B.; MAIA, A. H. N.; ALVES, R. E.; XAVIER, D. S.; MATOS, N. M. S.. Caracterização física de frutos do pequizeiro nativos da Chapada do Araripe-CE. Revista Brasileira de Fruticultura, Jaboticabal, v.31, n.4, p.1196-1201, 2009.

OLIVEIRA, M. E.; GUERA, N. B.; BARROS, L. M.; ALVES, R. E.. Aspectos agronômicos e de qualidade do Pequi. Fortaleza: Embrapa, 2008.

OLIVEIRA, M. N. S.; GUSMÃO, E.; LOPES, P. S. N.; SIMÕES, M. O. M.; RIBEIRO, L. M.; DIAS, B. A. S.. Estádio de maturação dos frutos e fatores relacionados aos aspectos nutritivos e de textura da polpa de pequi (Caryocar brasiliense Camb.). Revista Brasileira de Fruticultura, Jaboticabal, v.28, n.3, p.380-386, 2006.

PEIXOTO, A. R.. O pequi e a lavoura no Cerrado. In: PEIXOTO, A. R.. Plantas oleaginosas arbóreas. São Paulo: Nobel, 1973. p.197-226.

PESSOA, A. S.; PODESTÁ, R.; BLOCK, J. M.; FRANCESHI, E.; DARIVA, C.; LANZA, M.. Extractio of pequi (Caryocar coriaceum) pulp oil using subcritical propane: determination of process yield and fatty acid profile. Journal of Supercritical Fluids, v.101, p.95-103, 2015.

PINTO, V. L.; TOSTES, L. C. L.. Aspectos anatômicos e fitoquímicos do óleo fixo do mesocarpo e da semente de Caryocar villosum (Aubl.) Pers. (Piquiá) coletados no Município de Tartarugalzinho. Monografia (Graduação em Ciências Biológicas) - Universidade Federal do Amapá, Macapá, 2007.

RAMOS, K. M. C.; SOUZA, V. A. B.. Características físicas e químico-nutricionais de frutos de pequizeiro (Caryocar coriaceum Wittm.) em populações naturais da região MeioNorte do Brasil. Revista Brasileira de Fruticultura, Jaboticabal, v.33, n.2, p.500-508, 2011.

RIBEIRO, D. M.. Propriedades físicas, químicas e bioquímicas de pequi (Caryocar brasiliense Camb.) de diferentes regiões do Cerrado. Dissertação (Mestrado em Nutrição humana) - Universidade de Brasília, Brasília, 2011.

SHANLEY, P.; MEDINA, G.. Frutíferas e plantas úteis na vida amazônica. Belém: CIFOR, 2005.

SOARES JÚNIOR, M. S.; BASSINELLO, P. Z.; CALIARI, M.; REIS, R. C.; LACERDA, D. B. C. L.; KOAKUZU, S. N. Development and chemical characterization of flour obtained from the external mesocarp of 'pequizeiro' fruit. Ciência e Tecnologia de Alimentos, Campinas, v.30, n.4, p.949-954, 2010.

SOUZA, T. M. H.. Estudo do potencial do fruto do pequi (Caryocar villosum) para obtenção de óleo e síntese de biodiesel. Rio Branco: SBQ, 2005.

VERA, R.; SOUZA, E. R. B.; FERNANDES, E. P.; NAVES, R. V.; SOARES JÚNIOR, M. S.; CALIARI, M.; XIMENES, P. A.. Caracterização física e química de frutos do pequizeiro (Caryocar brasiliense Camb.) oriundos de duas regiões no estado de Goiás, Brasil. Pesquisa Agropecuária Tropical, Goiás, v.37, n.2, p.93-99, 2007.

VESTANO JÚNIOR, B.; BARBOSA, A. P.. Propagação vegetativa do piquiá (Caryocar villossum Pers.) por estaquia. Acta Amazônica, Manaus, v.13, p.143-148, 1983.

XAXIER, W. K. S.. Aproveitamento de recursos naturais da biodiversidade amapaense: óleo fixo de piquiá (Caryocar villosum (AUBL) PERS.) como anti-inflamatório tópico. Dissertação (Mestrado em Biodiversidade Tropical) Fundação Universidade Federal do Amapá, Macapá, 2011.

ZAR, J. H.. Biostatistical Analysis. New Jersey: Pretence Hall, 1999.

A CBPC - Companhia Brasileira de Produção Científica (CNPJ: 11.221.422/0001-03) detém os direitos materiais desta publicação. Os direitos referem-se à publicação do trabalho em qualquer parte do mundo, incluindo os direitos às renovações, expansões e disseminações da contribuição, bem como outros direitos subsidiários. Todos os trabalhos publicados eletronicamente poderão posteriormente ser publicados em coletâneas impressas sob coordenação da Sustenere Publishing, da Companhia Brasileira de Produção Científica e seus parceiros autorizados. Os (as) autores (as) preservam os direitos autorais, mas não têm permissão para a publicação da contribuição em outro meio, impresso ou digital, em português ou em tradução. 